\section{EQUAÇÃO DA DISPERSÃO DE POLUENTES EM RIOS, PARA DIFERENTES FONTES DE LANÇAMENTOS, CONSIDERANDO OS DIVERSOS PARÂMETROS HIDRÁULICOS DO CORPO HÍDRICO}

\author{
Ticiana Fontoura Vidal ${ }^{1}$ \\ Patrícia Freire Chagas ${ }^{2}$
}

Raimundo Oliveira de Souza ${ }^{3}$

\begin{abstract}
RESUMO
Para este trabalho, desenvolveu-se uma metodologia com base na mecânica dos fluidos computacional para avaliar o comportamento do transporte de poluentes em rios naturais, sujeitos a diferentes tipos de lançamentos. A metodologia empregada utiliza fundamentos da teoria de transporte de massa, avaliando os campos de concentração de substâncias poluentes, ao longo do tempo e do espaço, de modo a verificar a influência de parâmetros como declividades do fundo do canal e coeficiente de rugosidade no comportamento deste campo. Para tal, desenvolveu-se um programa computacional que permitiu a realização de um conjunto de simulações para vários tipos de cenários reais encontrados nas relações entre o homem e o rio. Os resultados mostraram que o comportamento da concentração é bastante influenciado pelas características hidráulicas do canal, quanto maior o coeficiente de rugosidade menor é a celeridade de propagação da nuvem poluente e para maiores declividades, maiores são os valores de concentração.
\end{abstract}

PALAVRAS-CHAVE: Transporte de Massa. Teoria Fuzzy.

\section{EQUATION OF DISPERSION OF POLLUTANTS IN RIVERS, FOR DIFFERENT RELEASES SOURCES WHEREAS THE OTHER HYDRAULIC PARAMETERS OF THE BODY WATER}

\begin{abstract}
For this work, we developed a methodology based on computational fluid mechanics to study the behavior of pollutant transport in natural rivers, subject to different types of releases. The methodology uses fundamentals of mass transfer theory, evaluating the concentration of pollutants fields over time and space in order to verify the influence of parameters such as the channel bottom slope and roughness coefficient in the behavior of this field. To this end, we developed a computer program that allowed for a set of simulations for various types of real scenarios found in the relationship between man and river. The results showed that the behavior of concentration is greatly influenced by the hydraulic characteristics of the channel, the higher the lower roughness coefficient is the speed of propagation of the pollutant cloud and higher slopes, the higher the concentration values.
\end{abstract}

KEYWORDS: Mass Transportation. Fuzzy Set Theory.

\footnotetext{
${ }^{1}$ Mestre em Engenharia Civil - Saneamento Ambiental, Universidade Federal do Ceará/Doutoranda em Engenharia Civil - Recursos Hídricos. ticianafvidal@yahoo.com.br

${ }^{2}$ Doutora em Recursos Hídricos, Universidade Federal do Ceará. pfchagas@yahoo.com.br

${ }^{3}$ Doutor em Hidráulica e Saneamento, Universidade Federal do Ceará/Professor Titular Departamento de Engenharia Hidráulica e Ambiental. rsouza@ufc.br.
} 


\section{ECUACIÓN DE LA CONTAMINACIÓN EN RÍOS DISPERSIÓN, DISTINTO DE LIBERACIÓN DE FUENTES DE AGUA MIENTRAS QUE LOS OTROS PARÁMETROS CORPORALES HIDRÁULICO}

\begin{abstract}
RESUMEN
Para este trabajo, hemos desarrollado una metodología basada en la mecánica de fluidos computacional para evaluar el comportamiento de transporte de contaminantes en los ríos naturales, sujetos a diferentes tipos de lanzamientos. La metodología utiliza fundamentos de la teoría de transferencia de masa, la evaluación de la concentración de los campos de contaminantes a través del tiempo y el espacio con el fin de verificar la influencia de parámetros tales como la pendiente del fondo del canal y coeficiente de rugosidad en el comportamiento de este campo. Para ello, hemos desarrollado un programa de ordenador que permitió una serie de simulaciones para diversos tipos de escenarios reales que se encuentran en las relaciones entre el hombre y el río. Los resultados mostraron que el comportamiento de la concentración está muy influenciada por las características hidráulicas de la canal, mayor es el coeficiente de rugosidad inferior es la velocidad de propagación de nube contaminante y para mayor pendiente, mayores son los valores de concentración
\end{abstract}

PALABRAS-CHAVE: Transito del massa. Teoría Fuzzy.

\section{INTRODUÇÃO}

Através dos estudos de qualidade de água é possível avaliar o comportamento de um campo de concentração em um sistema hídrico após o lançamento de uma carga poluente. Para tal, devem-se entender os fenômenos físicos, químicos e biológicos responsáveis pelo movimento e dispersão dos contaminantes nesse sistema. Os fenômenos físicos relacionados com a hidrologia são regidos por leis fundamentais da física, tais como: conservação de massa, leis da dinâmica newtoniana e leis da termodinâmica. Essas leis, quando aplicadas a meios contínuos, são representadas por equações diferenciais parciais que assumem diferentes formas, de acordo com o fenômeno a ser estudado (DIAS, 2003).

Os modelos matemáticos de qualidade de água são capazes de representar um processo físico qualquer através da formulação de um conjunto de equações matemáticas. Como todo sistema natural, os sistemas hídricos são bastante complexos e envolvem a interação entre diversos ramos da ciência - hidrologia, hidráulica, geomorfologia e transporte de massa. A diversidade de parâmetros que são necessários para descrever o ecossistema aquático, os processos físicos e a variabilidade espacial e temporal aumentam as dificuldades da modelagem. Algumas vantagens dessa modelagem são a velocidade de solução com o uso do 


\section{Periádica Eletrânica

computador; o seu alcance, permitindo a simulação de vários cenários para um mesmo problema e o fator custo que é bem menor do que o da modelagem física.

$\mathrm{Na}$ solução das equações que compõem um modelo empregam-se, normalmente, métodos analíticos ou métodos numéricos. A obtenção de soluções analíticas para equações diferenciais parciais, quando possível, é de fundamental importância, pois permite uma análise mais precisa, e também, em geral, revela os parâmetros adimensionais que controlam a solução do modelo.

Entretanto, o método analítico possui sérias limitações decorrentes da complexidade das equações diferenciais que, normalmente, definem o modelo. Essas equações, somente, tem soluções para específicas condições de contorno. Caso contrário, não existindo uma solução analítica, é necessário resolver a equação diferencial numericamente (SAIKI, 2006).

Com o enorme desenvolvimento computacional, as soluções numéricas tornaram-se técnicas altamente eficientes nos mais diversos campos da ciência. $\mathrm{Na}$ modelagem dos sistemas hídricos, por exemplo, tendo em vista a grande complexidade das equações que descrevem os fenômenos ligados ao escoamento, onde quase sempre não há soluções analíticas para o modelo, os métodos numéricos têm desempenhado um importante papel.

A metodologia proposta tem como princípio básico a determinação do comportamento do campo de concentração no rio natural, sujeito a diferentes cenários de lançamentos de efluentes, a fim de relacionar a declividade do fundo do canal e o coeficiente de rugosidade com a concentração, com o uso de um programa computacional especialmente desenvolvido para tal, na linguagem Fortran.

\section{METODOLOGIA}

O campo de concentração é obtido a partir da solução da equação diferencial da difusão advectiva. A seguir serão apresentadas as formulações pertinentes a este modelo.

1. Modelo de Transporte de Poluentes 
O campo de concentração é calculado mediante da equação da difusão advectiva definida por (JAMES, 1993):

(1)

$$
\frac{\partial C}{\partial t}+u \frac{\partial C}{\partial x}=\frac{1}{A} \frac{\partial}{\partial x}\left(A E \frac{\partial C}{\partial x}\right)-K C+S_{D}
$$

Onde:

C é a concentração da substância ao longo do canal, em $\mathrm{kg} / \mathrm{m}^{3}$;

u é a velocidade ao longo do canal, em $\mathrm{m} / \mathrm{s}$;

A é a área da seção transversal do canal, em $\mathrm{m}^{2}$;

E é o coeficiente de dispersão longitudinal;

$\mathrm{K}$ é o coeficiente de decaimento da substância, em $\mathrm{T}^{-1}$;

$S_{D}$ representa o lançamento distribuído ao longo do canal.

Desenvolvendo a equação da difusão advectiva, tem-se:

$$
\frac{\partial C}{\partial t}+u \frac{\partial C}{\partial x}=\frac{1}{A}\left[A E \frac{\partial^{2} C}{\partial x^{2}}+E \frac{\partial C}{\partial x} \frac{\partial A}{\partial x}+A \frac{\partial C}{\partial x} \frac{\partial E}{\partial x}\right]-K C+S_{D}
$$

(2)

Rearrumando a equação, tem-se:

$$
\frac{\partial C}{\partial t}+u \frac{\partial C}{\partial x}-\frac{E}{A} \frac{\partial A}{\partial x} \frac{\partial C}{\partial x}-\frac{\partial E}{\partial x} \frac{\partial C}{\partial x}=E \frac{\partial^{2} C}{\partial x^{2}}-K C+S_{D}
$$

(3)

Colocando o termo $\frac{\partial C}{\partial x}$ em evidência, resulta em:

$$
\frac{\partial C}{\partial t}+\left[u-\frac{E}{A} \frac{\partial A}{\partial x}-\frac{\partial E}{\partial x}\right] \frac{\partial C}{\partial x}=E \frac{\partial^{2} C}{\partial x^{2}}-K C+S_{D}
$$

(4)

$$
\begin{aligned}
& \text { Fazendo } \psi=\left[u-\frac{E}{A} \frac{\partial A}{\partial x}-\frac{\partial E}{\partial x}\right], \text { a equação fica: } \\
& \frac{\partial C}{\partial t}+\psi \frac{\partial C}{\partial x}=E \frac{\partial^{2} C}{\partial x^{2}}-K C+S_{D}
\end{aligned}
$$


O coeficiente de dispersão longitudinal pode ser calculado através da equação (Fischer,1979):

$$
E=0.05937 \frac{Q}{S_{0} B}
$$

Onde:

$Q$ é a vazão, em $\mathrm{m}^{3} / \mathrm{s}$;

$\mathrm{S}_{0}$ é a declividade longitudinal do rio;

B é a largura média do canal principal.

2. Esquema Numérico para a Solução do Modelo de Transporte

Tendo em vista a natureza da equação da difusão advectiva, o esquema numérico baseado no Método das Diferenças Finitas foi utilizado para resolver a equação de transporte. Este método permite a aproximação das derivadas parciais de forma explícita ou implícita. Para este trabalho utilizou-se o esquema implícito, ou Método de Crank-Nicolson. A razão para tal escolha baseia-se no fato de que este tipo de discretização é tido como um exemplo de esquema numérico estável e recomendado sempre que opções específicas não estiverem disponíveis (CUMINATO \& OISHI, 2011).

\section{RESULTADOS}

Para avaliar a eficiência do programa computacional com relação ao comportamento da concentração, comparou-se a solução numérica obtida pelo programa desenvolvido com a solução analítica proposta por Chapra (1997). Essa simulação foi feita considerando um rio urbano com comprimento de $2000 \mathrm{~m}$, largura do canal $5 \mathrm{~m}$, declividade de fundo de $0,0005 \mathrm{~m} / \mathrm{m}$, coeficiente de rugosidade de Manning de 0,0138. A malha $x$-t foi dividida em 30 trechos de $100 \mathrm{~m}$ de comprimento, na direção $x$ da corrente, e em 32 intervalos de tempo de 1,5 min, totalizando um tempo máximo de $0,8 \mathrm{~h}$.

Pela Figura 1, verificou-se que os valores encontrados através da modelagem matemática proposta, mostraram uma excelente concordância com a solução analítica apresentada por Chapra (1997). 
Figura 1 - Comparação da distribuição da concentração pela solução numérica, obtida pelo modelo desenvolvido e pela solução analítica proposta por Chapra (1997).

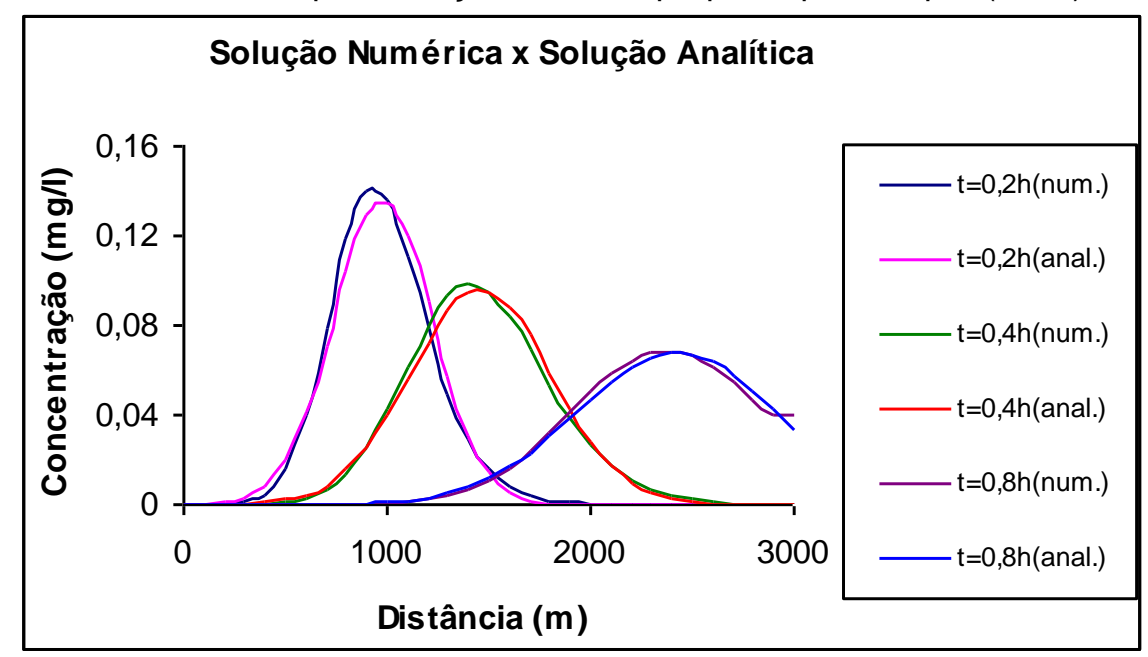

Diante desta concordância de resultados, iniciou-se a fase de simulações para o estudo da concentração ao longo do rio. Dentre os principais cenários definidos, pode-se destacar o lançamento de uma carga puntiforme instantânea para uma substância conservativa; propagação de uma onda despoluída que chega num rio poluído; verificação do decaimento de uma substância não conservativa e do lançamento de uma carga puntiforme e, por último, a análise de um cenário com uma carga difusa para substâncias com e sem decaimento.

Nas simulações seguintes, considerou-se um canal retangular com comprimento de $50.000 \mathrm{~m}$, que foi discretizado em 50 trechos de $1000 \mathrm{~m}$ de comprimento cada, na direção longitudinal do rio. Com relação ao tempo foram definidos 500 intervalos de tempo de $150 \mathrm{~s}$, perfazendo um tempo máximo de, aproximadamente, 21 horas. A vazão inicial do rio é de $50 \mathrm{~m}^{3} / \mathrm{s}$. Os valores da declividade do canal, da rugosidade e da vazão inicial são definidos de acordo com a simulação desejada.

A simulação numérica mostrada na Figura 2 analisa a distribuição da concentração ao longo do tempo, após o lançamento instantâneo de uma substância poluente com concentração igual a $200 \mathrm{mg} / \mathrm{L}$, no ponto $x$ igual a $5 \mathrm{~km}$ da seção de referência do canal. Considerou-se o canal com declividade igual a $0,0001 \mathrm{~m} / \mathrm{m}$, rugosidade igual a 0,06 e concentração inicial igual a 10mg/L. 
Figura 2 - Distribuição da concentração para o lançamento de uma carga pontual, $200 \mathrm{mg} / \mathrm{L}$, lançada no ponto $x=5 \mathrm{~km}$.

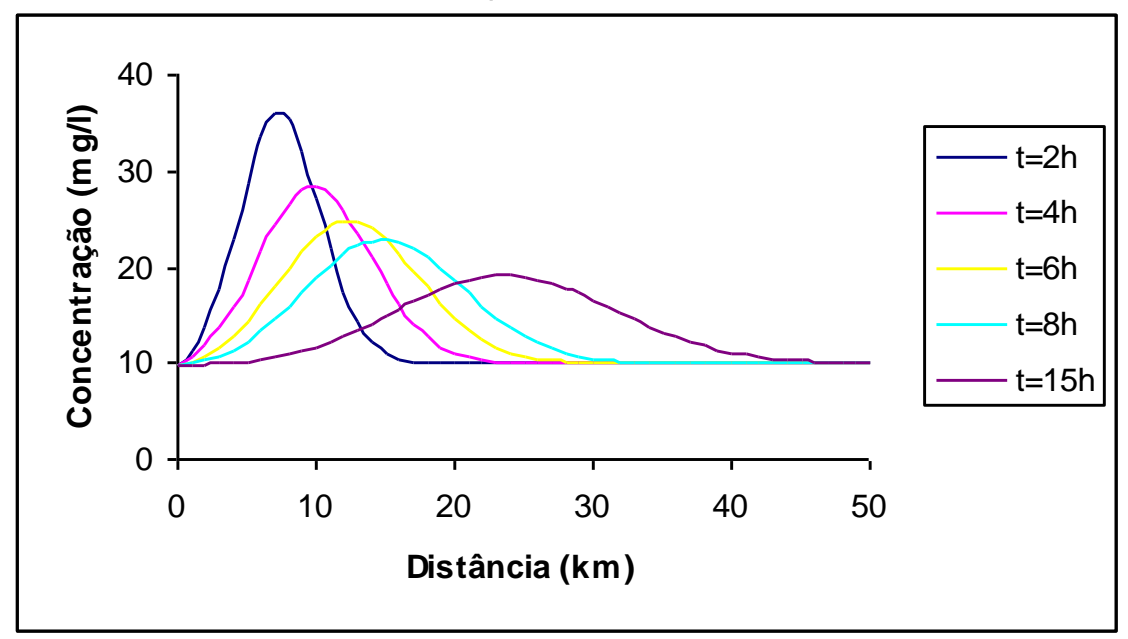

Pode-se verificar através da figura, não só o efeito do processo advectivo, conduzindo a nuvem poluente rio abaixo, como também o efeito difusivo, causado pelo coeficiente de difusão. Este coeficiente faz com que as larguras dos perfis de concentração aumentem com o tempo. Observou-se ainda, o processo de redução dos picos de concentração, ao longo do tempo. Estes resultados são semelhantes aos resultados obtidos a partir da solução analítica da equação da difusão advectiva.

A Figura 3 mostra o resultado da simulação, para $S_{0}=0,0001 \mathrm{~m} / \mathrm{m}$ e $\mathrm{n}=0,06$. Neste caso o cenário proposto induz à presença de uma condição de contorno à montante cuja concentração é igual a zero.

Figura 3 - Distribuição da concentração para uma condição de contorno a montante cuja concentração é igual a zero.

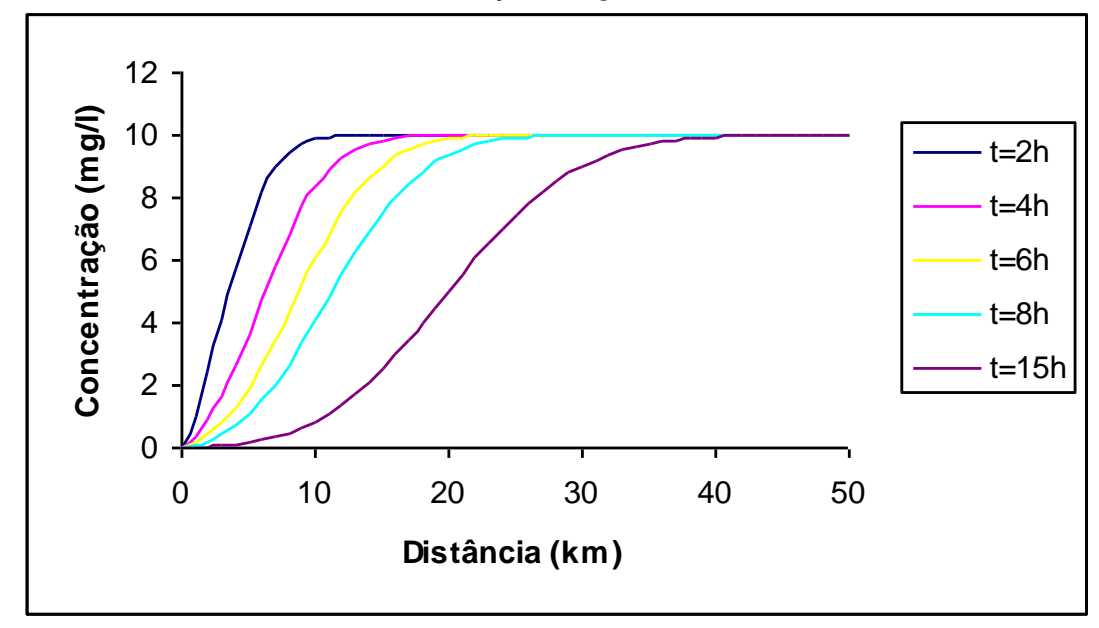


Verificou-se a evolução do processo de despoluição do rio no tempo, para diferentes seções. Para um tempo de 15 horas a concentração inicial do rio, de 10 $\mathrm{mg} / \mathrm{L}$, só aparece depois de $35 \mathrm{~km}$. Isto quer dizer, que as concentrações nos pontos anteriores a este estão com valores menores.

$\mathrm{Na}$ Figura 4, com $\mathrm{S}_{0}=0,0001 \mathrm{~m} / \mathrm{m}$ e $\mathrm{n}=0,06$, considerou-se o lançamento contínuo de uma substância poluente com concentração de $200 \mathrm{mg} / \mathrm{L}$ que, ao diluirse com a vazão do rio, resultou numa concentração final de entrada, em $x=0$, de 5,8 $\mathrm{mg} / \mathrm{L}$. Verifica-se que ao longo do tempo a concentração do rio tende a se estabilizar em $5,8 \mathrm{mg} / \mathrm{L}$.

Figura 4 - Distribuição da concentração para um lançamento contínuo na origem.

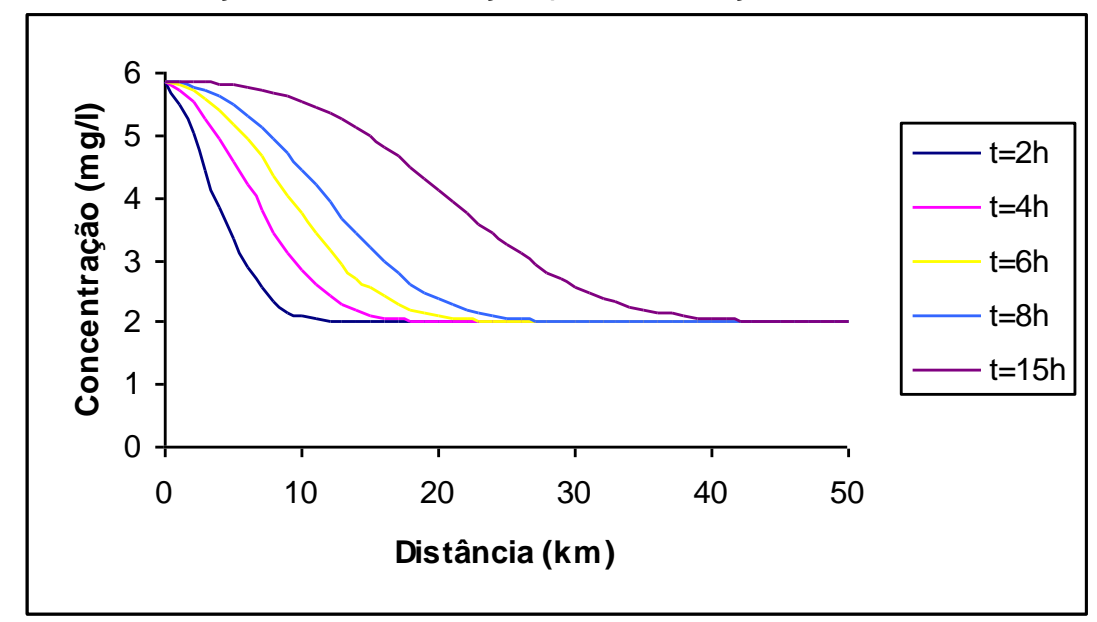

A Figura 5 apresenta os resultados obtidos a partir de um cenário um pouco diferente do anterior, mas com $\mathrm{n}=0,06 ; \mathrm{S}_{0}=0,0001 \mathrm{~m} / \mathrm{m}$ e $\mathrm{C}_{0}=10 \mathrm{mg} / \mathrm{L}$. Nesta simulação, avalia-se a influência da propagação de uma onda dinâmica com concentração $C_{0} / 2$, onde $C_{0}$ é a concentração inicial do rio. Neste caso, verificou-se que a propagação de uma onda de cheia ao longo do rio influência na capacidade de diluição do mesmo, criando assim um trem de ondas de despoluição em fase com a onda de cheia. 
Figura 5 - Distribuição da concentração sob o efeito da entrada de uma onda de cheia com C=5mg/L, em um canal com $\mathrm{C}_{0}=10 \mathrm{mg} / \mathrm{L}$.

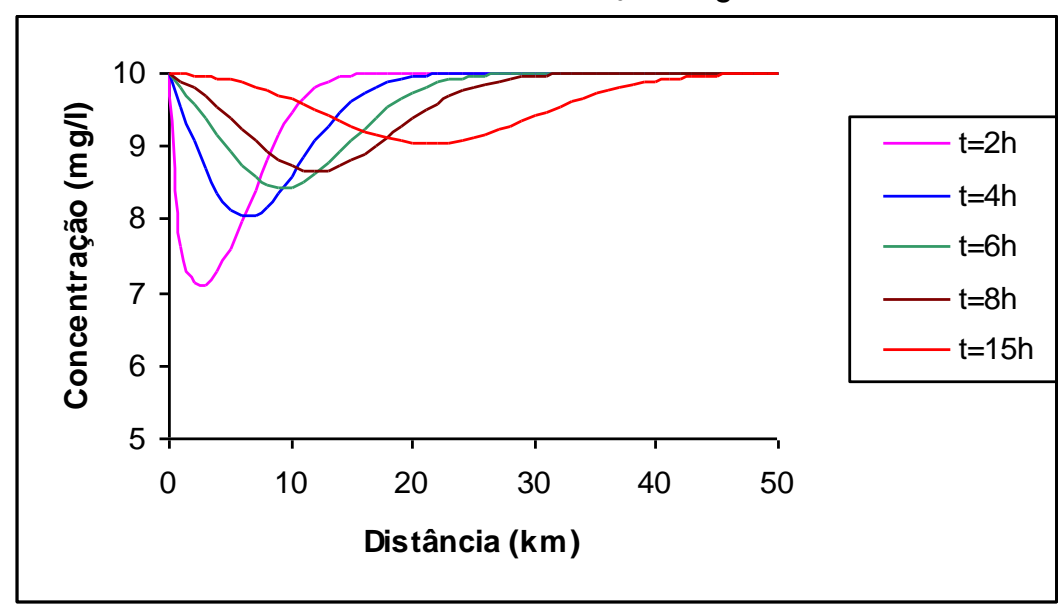

A Figura 6, para $\mathrm{n}=0,06$ e $\mathrm{S}_{0}=0,0001 \mathrm{~m} / \mathrm{m}$, mostra a distribuição da concentração para um cenário no qual uma substância não conservativa é lançada em um canal. A condição de contorno da concentração é igual a $10 \mathrm{mg} / \mathrm{L}$ e a taxa de decaimento usada foi de 0,0001/T, com $\mathrm{T}$ em seg. Assim, verificou-se que 0 processo de decaimento é significativo para a concentração, tendo a mesma seus valores reduzidos ao longo do tempo. Pode-se verificar que esses resultados estão de acordo com a solução analítica da equação da difusão advectiva para uma substância não conservativa.

Figura 6 - Distribuição da concentração ao longo do canal para uma substância não conservativa.

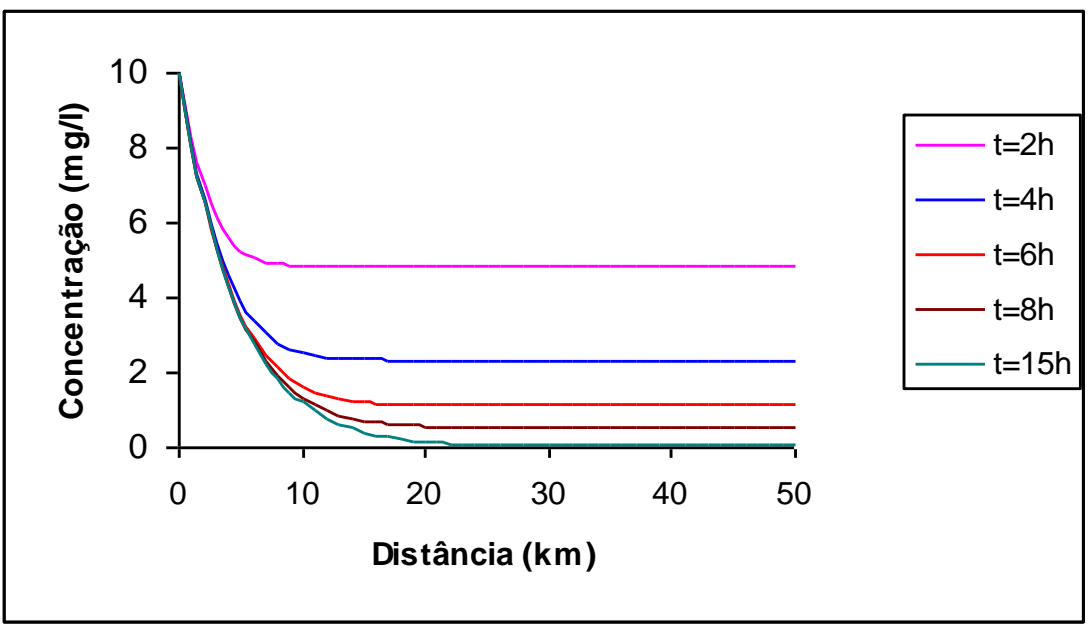

A simulação da Figura 7 mostra a distribuição da concentração em 3 seções diferentes do canal, $x$ igual a 10, 15 e $20 \mathrm{~km}$, após o lançamento pontual de 400 $\mathrm{mg} / \mathrm{L}$, em $\mathrm{x}=5 \mathrm{~km}$. A concentração inicial do canal é de $2 \mathrm{mg} / \mathrm{L}$, a rugosidade é igual a 0,05 e a declividade é igual a $0,0001 \mathrm{~m} / \mathrm{m}$. 
Figura 7 - Distribuição da concentração ao longo do tempo para um lançamento pontual em $x=5 \mathrm{~km}$.

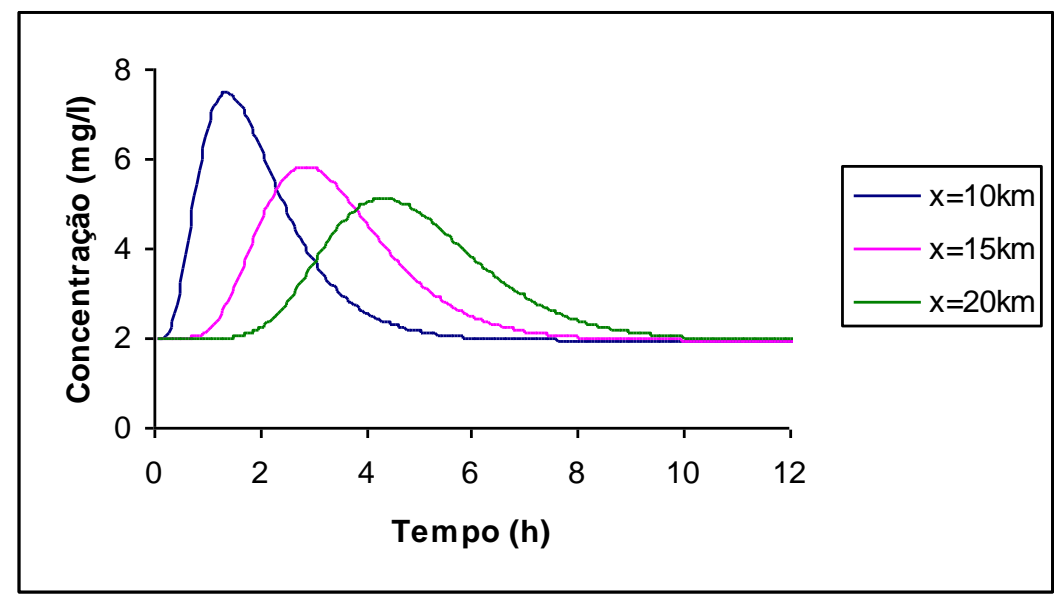

Na Figura 7, percebe-se que há uma diminuição, temporal e espacial, dos níveis de concentração, onde os valores máximos são 7,5; 6,0; 5,0 mg/l, para x igual a 10, 15 e $20 \mathrm{~km}$ e ocorrem para t igual a 1:30, 3:00 e 4:30 horas. Verifica-se que quanto mais distante a seção maior o tempo para a chegada do pico da onda de poluição. Observa-se, também, que a taxa de redução do pico de concentração é mais intensa no início do processo ficando mais suave com o passar do tempo. Este resultado está de acordo com os princípios que governam os processos de transporte de massa. Segundo a Lei de Fick, o fluxo de massa entre duas seções qualquer de um domínio é proporcional ao gradiente de concentração entre as duas seções. Neste caso, o gradiente de concentração é maior no início do processo.

Nas Figuras 8 e 9, verifica-se a distribuição da concentração para 0 lançamento de uma carga difusa, com $S_{D}=0,0005 \mathrm{mg} / \mathrm{m} / \mathrm{s}$, ao longo de todo o canal. A concentração inicial é igual a zero, a rugosidade é igual a 0,05 e a declividade é igual a $0,0001 \mathrm{~m} / \mathrm{m}$. Entretanto, essas simulações são diferentes porque na primeira, a substância considerada é conservativa, enquanto que na segunda a substância é não conservativa com decaimento igual a 0,0001/T. 
Figura 8 - Distribuição da concentração para o lançamento de uma carga difusa ao longo do canal para uma substância conservativa.

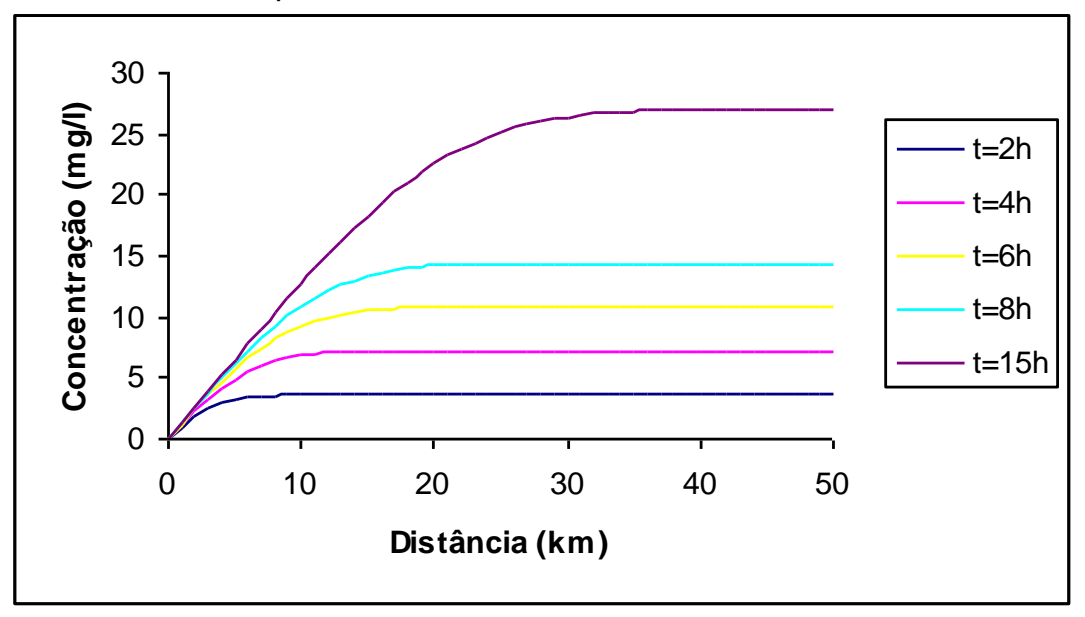

Como na Figura 8 não há decaimento, verifica-se que para um tempo igual a 15 horas o valor da concentração chega próximo de $30 \mathrm{mg} / \mathrm{L}$. Já para a Figura 9, a influência do decaimento faz com que a concentração para o mesmo tempo não ultrapasse à $5 \mathrm{mg} / \mathrm{L}$.

Figura 9 - Distribuição da concentração para o lançamento de uma carga difusa ao longo do canal para uma substância não conservativa com K=0,0001/T.

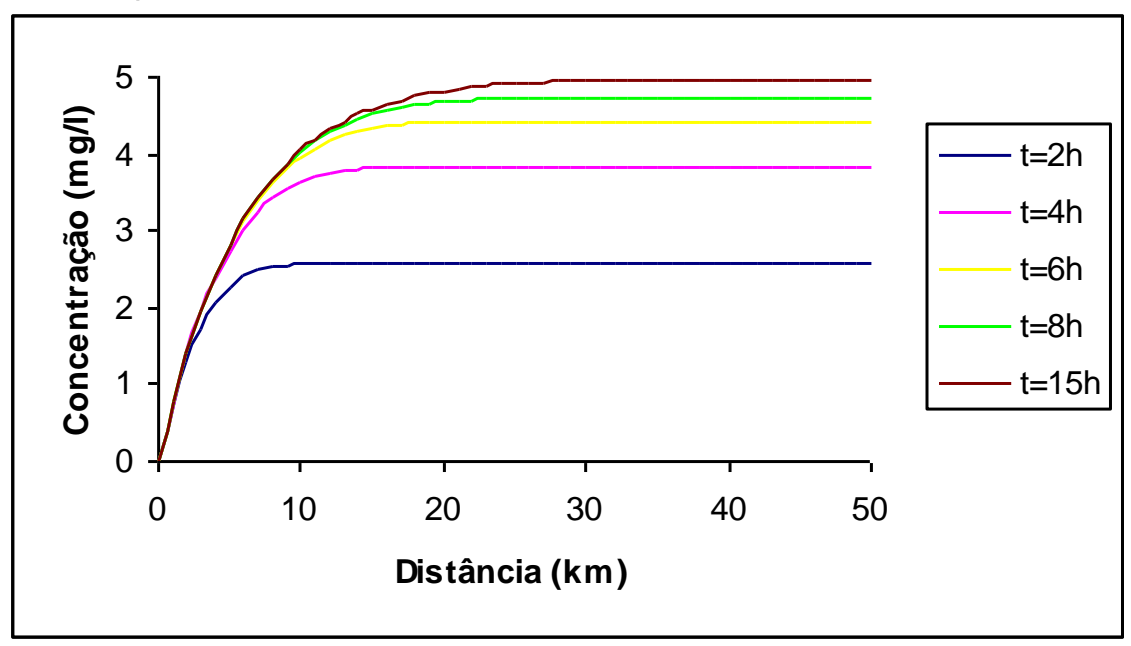

A Figura 9 mostra, ainda que, para longos tempos de observação, a concentração se estabiliza no valor $S_{D} / K$, onde $S_{D}$ representa a taxa de lançamento lateral, ou difuso, e $\mathrm{K}$ o de decaimento. Este resultado está perfeitamente de acordo com os resultados obtidos a partir da solução analítica da equação da difusão advectiva, mostrando, portanto, a capacidade do modelo em estudo. 


\section{Periódica Eletranica

\section{CONCLUSÕES}

Os resultados obtidos permitiram concluir que o comportamento da concentração é bastante influenciado pelas características hidráulicas do canal. Neste caso, a rugosidade afeta a celeridade de propagação da nuvem poluente, mas este parâmetro não tem tanta influência na amplitude da mesma. Ou seja, quanto maior o coeficiente de rugosidade menor é a celeridade de propagação da nuvem poluente. Já para o caso da declividade os resultados mostraram uma significativa influência deste parâmetro no desenvolvimento do perfil de concentração. Para maiores declividades, maiores são os valores da concentração. Também foi possível verificar que a entrada de uma onda de cheia com baixas concentrações, num rio poluído, provoca uma onda de despoluição com as mesmas características da propagação da onda de cheia.

\section{REFERÊNCIAS}

CHAPRA, S. C. Surface Water-Quality Modeling. New York: McGraw-Hill, 1997, 844p.

CUMINATO, J. A \& OISHI, C. M. Estabilidade do método de Crank-Nicolson para as equações de Navier-Stokes. I Congresso de Matemática Aplicada e Computacional, 2011.

DIAS, N. L. Obtenção de uma Solução Analítica da Equação de Difusão-Advecção com decaimento de $1^{\text {a }}$ ordem pelo Método da Transformação de Similaridade Generalizada. Revista Brasileira de Recursos Hídricos, v. 8, n.1, p. 181-188, 2003.

FISCHER, H. B. Mixing in Inland and Coastal Water, Academic Press, Inc, 1979.

JAMES, A. An Introduction to Water Quality Modelling. 2nd Edition. By John Wiley \& Sons Ltd, 1993, 311p.

SAIKI, M. E. Equação da onda unidimensional: um estudo analítico e numérico. Monografia (Graduação) - Universidade Católica de Brasília, Brasília, 2006. 15p. 